\title{
Gamma Decay of Unbound Neutron-Hole States in ${ }^{133} \mathrm{Sn}$
}

\author{
V. Vaquero, ${ }^{1}$ A. Jungclaus, ${ }^{1,}$ P. Doornenbal, ${ }^{2}$ K. Wimmer, ${ }^{3}$ A. Gargano, ${ }^{4}$ J. A. Tostevin, ${ }^{5}$ S. Chen, ${ }^{2,6}$ E. Nácher, ${ }^{1}$ E. Sahin, ${ }^{7}$ \\ Y. Shiga, ${ }^{8}$ D. Steppenbeck, ${ }^{2}$ R. Taniuchi, ${ }^{2,3}$ Z. Y. Xu, ${ }^{9}$ T. Ando, ${ }^{3}$ H. Baba, ${ }^{2}$ F. L. Bello Garrote, ${ }^{7}$ S. Franchoo, ${ }^{10}$ \\ K. Hadynska-Klek, ${ }^{7}$ A. Kusoglu, ${ }^{11,12}$ J. Liu, ${ }^{9}$ T. Lokotko, ${ }^{9}$ S. Momiyama, ${ }^{3}$ T. Motobayashi, ${ }^{2}$ S. Nagamine, ${ }^{3}$ N. Nakatsuka, ${ }^{13}$ \\ M. Niikura, ${ }^{3}$ R. Orlandi, ${ }^{14}$ T. Saito, ${ }^{3}$ H. Sakurai, ${ }^{2,3}$ P. A. Söderström, ${ }^{2}$ G. M. Tveten, ${ }^{7}$ Zs. Vajta, ${ }^{15}$ and M. Yalcinkaya ${ }^{11}$ \\ ${ }^{1}$ Instituto de Estructura de la Materia, CSIC, E-28006 Madrid, Spain \\ ${ }^{2}$ RIKEN Nishina Center, 2-1 Hirosawa, Wako, Saitama 351-0198, Japan \\ ${ }^{3}$ Department of Physics, University of Tokyo, 7-3-1 Hongo, Bunkyo, Tokyo 113-0033, Japan \\ ${ }^{4}$ Istituto Nazionale di Fisica Nucleare, Complesso Universitario di Monte S. Angelo, I-80126 Napoli, Italy \\ ${ }^{5}$ Department of Physics, University of Surrey, Guildford, Surrey GU2 7XH, United Kingdom \\ ${ }^{6}$ School of Physics and State Key Laboratory of Nuclear Physics and Technology, Peking University, \\ Bejing 100871, People's Republic of China \\ ${ }^{7}$ Department of Physics, University of Oslo, NO-0316 Oslo, Norway \\ ${ }^{8}$ Department of Physics, Rikkyo University, Tokyo, Japan \\ ${ }^{9}$ Department of Physics, The University of Hong Kong, Pokfulam, Hong Kong \\ ${ }^{10}$ Institut de Physique Nucléaire Orsay, IN2P3-CNRS, 91406 Orsay Cedex, France \\ ${ }^{11}$ Department of Physics, Faculty of Science, Istanbul University, Vezneciler/Fatih, 34134 Istanbul, Turkey \\ ${ }^{12}$ ELI-NP, Horia Hulubei National Institute of Physics and Nuclear Engineering, 077125 Magurele, Romania \\ ${ }^{13}$ Department of Physics, Faculty of Science, Kyoto University, Kyoto 606-8502, Japan \\ ${ }^{14}$ Advanced Science Research Center, Japan Atomic Energy Agency, Tokai, Ibaraki 319-1195, Japan \\ ${ }^{15}$ MTA Atomki, P.O. Box 51, Debrecen H-4001, Hungary
}

(Received 28 February 2017; published 17 May 2017)

\begin{abstract}
Excited states in the nucleus ${ }^{133} \mathrm{Sn}$, with one neutron outside the double magic ${ }^{132} \mathrm{Sn}$ core, were populated following one-neutron knockout from a ${ }^{134} \mathrm{Sn}$ beam on a carbon target at relativistic energies at the Radioactive Isotope Beam Factory at RIKEN. Besides the $\gamma$ rays emitted in the decay of the known neutron single-particle states in ${ }^{133} \mathrm{Sn}$ additional $\gamma$ strength in the energy range 3.5-5.5 MeV was observed for the first time. Since the neutron-separation energy of ${ }^{133} \mathrm{Sn}$ is low, $S_{n}=2.402(4) \mathrm{MeV}$, this observation provides direct evidence for the radiative decay of neutron-unbound states in this nucleus. The ability of electromagnetic decay to compete successfully with neutron emission at energies as high as $3 \mathrm{MeV}$ above threshold is attributed to a mismatch between the wave functions of the initial and final states in the latter case. These findings suggest that in the region southeast of ${ }^{132} \mathrm{Sn}$ nuclear structure effects may play a significant role in the neutron versus $\gamma$ competition in the decay of unbound states. As a consequence, the common neglect of such effects in the evaluation of the neutron-emission probabilities in calculations of global $\beta$-decay properties for astrophysical simulations may have to be reconsidered.
\end{abstract}

DOI: 10.1103/PhysRevLett.118.202502

The atomic nucleus offers a unique opportunity to study the competition between three of the four fundamental forces known to exist in nature, namely, the strong nuclear, the electromagnetic, and the weak nuclear interaction. In general, the decay of an excited nuclear state follows the hierarchy of these forces. The emission of one or more particles mediated by the strong interaction dominates the decay of unbound states while bound excited states usually decay by electromagnetic radiation until the ground state is reached. Finally, the latter decays via $\beta$ decay mediated by the weak nuclear interaction. The different strengths of these interactions are reflected by the time scales of the above mentioned processes, ranging from typically $10^{-22} \mathrm{~s}$ for fast particle decays to $10^{7} \mathrm{~s}$ for slow $\beta$ decays and $10^{30} \mathrm{~s}$ for double $\beta$ decays. Of course there are exceptions to this general rule. There are many cases known in which $\beta$ decay wins against electromagnetic decay because the latter requires the emission of a $\gamma$ ray of high multipolarity and/or low energy. In the case of the strong force the Coulomb barrier can defer the emission of charged particles and allow electromagnetic decay to compete above the separation energy. In the case of neutron emission on the neutron-rich side of the nuclear chart, in the absence of a Coulomb barrier, only the angular momentum barrier may hinder the neutron emission and thus favor $\gamma$ decay. Indeed $\gamma$-decaying high-spin states above the neutron-separation energy have been identified in several neutron-rich nuclei. In general, however, it is assumed that neutron decay dominates for all low- to moderate-spin states above the neutron threshold. This assumption is commonly used in theoretical calculations of global $\beta$-decay properties that are employed for astrophysical calculations, for example, for 
the description of the rapid neutron-capture process ( $r$ process) of nuclear synthesis.

However, according to Fermi's golden rule the probability of a certain decay process occuring does not only depend on the strength of the interaction and the density of final states, but also on the overlap between the wave functions of the parent and daughter states [1]. By means of this latter ingredient, nuclear structure effects can influence decay rates and thus have an impact, for example, on the competition between neutron emission and $\gamma$ deexcitation above the neutron-separation energy in neutron-rich nuclei. In recent years several cases have been reported in which electromagnetic decay successfully competes with neutron emission in the decay of unbound states with excitation energies up to more than $2 \mathrm{MeV}$ above the neutronseparation energy [2-5], i.e., well beyond the first few hundred $\mathrm{keV}$ where neutron emission is hindered by the low penetrability. In some of these works nuclear structure arguments based on theoretical calculations have been put forward to explain the experimental findings. On the neutron-deficient side of the nuclear chart, the $\gamma$ decay of isobaric analog states far above the proton-separation energy has been observed in $f p$ shell nuclei and explained by the fact that proton emission is isospin forbidden in these cases $[6,7]$.

In this Letter we investigate the nuclear structure aspect in the decay of unbound states by means of a very simple nuclear system, namely, the nucleus ${ }^{133} \mathrm{Sn}$. This nucleus has only one valence neutron in the $N=82-126$ major shell outside ${ }^{132} \mathrm{Sn}$, which is generally considered as a very robust double magic core. Neutron single-particle energies (SPE) of $854,1367,1561$, and $2002 \mathrm{keV}$ for the $2 p_{3 / 2}$, $2 p_{1 / 2}, 0 h_{9 / 2}$, and $1 f_{5 / 2}$ orbitals, respectively, relative to the $1 f_{7 / 2}$ orbital, have been established combining the information from both $\beta$ decay and $(d, p)$ neutron-transfer experiments [8-11]. For the $0 i_{13 / 2}$ SPE an energy range of 2360-2600 keV has recently been proposed based on the systematics of $13 / 2^{+}$levels in $N=83$ nuclei in comparison with shell-model calculations [12]. The neutron singlehole states in ${ }^{133} \mathrm{Sn}$ are expected at excitation energies far above $S_{n}$. In Ref. [8], a line at $1.26 \mathrm{MeV}$ in the neutron spectrum measured following the $\beta$ decay of ${ }^{134}$ In has tentatively been assigned to the decay of the $0 h_{11 / 2}^{-1}$ hole state in ${ }^{133} \mathrm{Sn}$, positioning this state at an excitation energy of around 3.66 MeV $\left[S_{n}=2.402(4) \mathrm{MeV}\right.$ [13]]. The present work reports on the study of the $\gamma$ decay of excited states in ${ }^{133} \mathrm{Sn}$ populated via one-neutron knockout from ${ }^{134} \mathrm{Sn}$ at relativistic energies.

The experiment was performed at the Radioactive Isotope Beam Factory at RIKEN. Secondary radioactive beams were produced via projectile fission of a $345 \mathrm{MeV} / \mathrm{u}{ }^{238} \mathrm{U}$ beam with an average intensity of $15 \mathrm{pnA}$, impinging on a 4-mm thick Be target. The ions of interest were separated from other reaction products and identified on an ion-by-ion basis by the BigRIPS in-flight separator [14]. The particle identification was performed using the $\Delta E$-TOF- $B \rho$ method in which the energy loss $(\Delta E)$, time of flight (TOF) and magnetic rigidity $(B \rho)$ are measured and used to determine the atomic number $Z$ and the mass-to-charge ratio $A / q$ of the fragments [15]. The identified ${ }^{134} \mathrm{Sn}$ ions then impinged with a kinetic energy of $165 \mathrm{MeV} / \mathrm{u}$ on a 3-mm thick $\mathrm{C}$ target. Reaction products created via nucleon removal were identified in the ZeroDegree (ZD) spectrometer [14], employing again the $\Delta E$-TOF- $B \rho$ method. Total reaction cross sections for the removal of $x$ neutrons, $\sigma_{x n}$, were determined from the yield of the respective reaction products and the number of incoming projectile ions taking into account the transmission through the $\mathrm{ZD}$ spectrometer, losses due to reactions with detector material along the beam line, and the properties of the $\mathrm{C}$ target. To detect $\gamma$ radiation emitted from excited reaction residues the secondary target was surrounded by the DALI2 spectrometer [16]. DALI2 consisted of $186 \mathrm{NaI}(\mathrm{Tl})$ detectors, covering polar angles in the range from $20^{\circ}$ to $150^{\circ}$, and had a photo peak efficiency of $15 \%$ for the $1.33-\mathrm{MeV} \gamma$ ray emitted by the stationary ${ }^{60} \mathrm{Co}$ source.

Figure 1 shows the Doppler-corrected $\gamma$-ray spectrum measured in coincidence with ${ }^{134} \mathrm{Sn}$ ions detected in BigRIPS and ${ }^{133} \mathrm{Sn}$ nuclei detected in the ZD spectrometer. The three lines at energies of 854, 1561, and $2002 \mathrm{keV}$ correspond to the decays of the $2 p_{3 / 2}, 0 h_{9 / 2}$, and $1 f_{5 / 2}$ single-particle states to the $1 f_{7 / 2}$ ground state while the 513-keV $\gamma$ ray depopulates the $2 p_{1 / 2}$ state at $1367 \mathrm{keV}$ to the $2 p_{3 / 2}$ level at $854 \mathrm{keV}$ [11]. Besides these known $\gamma$ rays, clearly additional $\gamma$ strength is observed above the

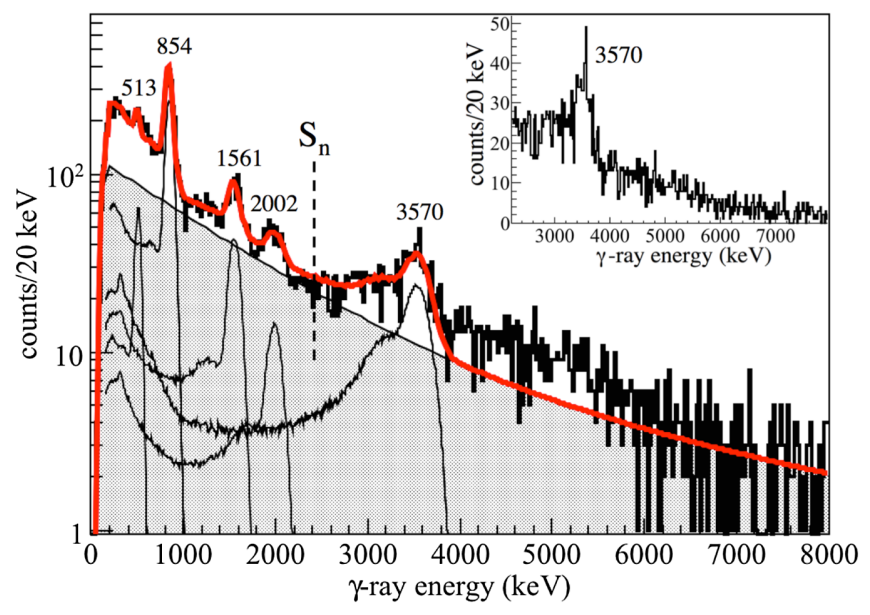

FIG. 1. Doppler-corrected $\gamma$-ray spectrum (for $\gamma$-ray multiplicity $M_{\gamma}=1$ after add back) of ${ }^{133} \mathrm{Sn}$ populated via one-neutron knockout from ${ }^{134} \mathrm{Sn}$. The response function fit to the experimental spectrum is shown by the thick red line while the individual components are shown as thin black lines. The background is indicated as the gray area. The inset shows the high-energy region of the spectrum on a linear scale. 
neutron separation energy, reaching up to about $5.5 \mathrm{MeV}$. To describe the experimental spectrum the response of the DALI2 array to the incident $\gamma$ radiation was simulated using GEANT4 [17]. In these simulations the precise $\gamma$-ray energies determined in Ref. [11] were employed. To increase the detection efficiency for high-energy $\gamma$ rays and to improve the peak-to-total ratio over the full energy range an addback algorithm was applied. All energy depositions registered in NaI crystals within a range of $15 \mathrm{~cm}$ from the center of the crystal with the highest energy signal were summed. Doppler correction was performed using the midtarget velocity of $\beta=0.497$. The background in the spectrum shown in Fig. 1 has been parametrized by the sum of two exponential functions cut off at low energy with an error function. Up to the neutron separation energy the experimental spectrum is well described by the sum of the background and the DALI2 response to the listed $\gamma$ rays. For the 854-keV line, however, a small shift with respect to the nominal energy was observed when only the most forward crystals of DALI2 were considered as shown in Fig. 2(a). This shift points to a lifetime of a few tens of ps for the 854-keV state because in that case the $\gamma$ ray emission takes place at a lower average recoil velocity than assumed in the simulation. Comparing the experimental line shapes obtained for two different angular ranges of DALI2 detectors to simulations, a value of $\tau=30(15)$ ps was deduced for the state at an excitation energy of $854 \mathrm{keV}$ (see Fig. 2). From this lifetime a transition strength of $B(E 2)=1.6(8)$ W.u. is calculated for the $2 p_{3 / 2} \rightarrow 1 f_{7 / 2}$ $E 2$ transition in ${ }^{133} \mathrm{Sn}$. This strength is comparable to that of the $2 d_{5 / 2} \rightarrow 1 g_{9 / 2}$ and $3 s_{1 / 2} \rightarrow 2 d_{5 / 2}$ neutron single-particle transitions in ${ }^{209} \mathrm{~Pb}[B(E 2)=2.5(7)$ and $B(E 2)=2.13(8)$ W.u., respectively, [18]]. Coming back to the spectrum shown in Fig. 1, the strongest peak above $S_{n}$ is well described by the DALI2 response to a single $\gamma$ ray with an energy of 3570(50) $\mathrm{keV}$. With respect to the $\gamma$ strength at even higher energy, unfortunately the limited statistics and poor energy resolution prohibit a more detailed analysis.

To interpret the $\gamma$-ray spectrum of ${ }^{133} \mathrm{Sn}$, the reaction process that led to the population of excited states in this
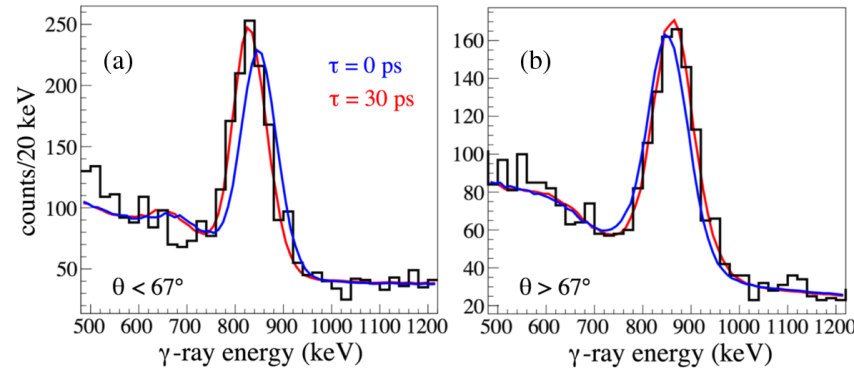

FIG. 2. Comparison between the experimental shape of the 854-keV line observed in the DALI2 detectors at polar angles (a) $<67^{\circ}$ and (b) $>67^{\circ}$ and simulations assuming lifetimes of $\tau=0$ (blue) and $\tau=30 \mathrm{ps}$ (red) for the $854-\mathrm{keV}$ state. nucleus has to be considered. In principle, the neutron removal can proceed from any neutron orbital that is occupied in the ground state of the projectile nucleus, in this case ${ }^{134} \mathrm{Sn}$. Considering the single-particle energies in ${ }^{133} \mathrm{Sn}$ it is expected that the valence-neutron pair in ${ }^{134} \mathrm{Sn}$ occupies dominantly the $1 f_{7 / 2}$ orbital. A knockout from this orbital results in the ground state of ${ }^{133} \mathrm{Sn}$ and, consequently, no $\gamma$ ray is emitted in this case. However, also the other neutron orbitals of the $N=82-126$ shell contribute to the composition of the wave function; i.e., there is a certain probability for the neutron pair to occupy the $2 p_{3 / 2}, 2 p_{1 / 2}, 0 h_{9 / 2}, 1 f_{5 / 2}$, and $0 i_{13 / 2}$ orbitals. Shellmodel calculations using realistic effective interactions [19] predict an $80 \%$ probability for the valence-neutron pair to occupy the $1 f_{7 / 2}$ orbital and contributions between $1.6 \%$ and $5.2 \%$ for all other orbitals of the $N=82-126$ shell. One-neutron knockout from the $2 p_{3 / 2}, 2 p_{1 / 2}, 0 h_{9 / 2}$, and $1 f_{5 / 2}$ orbitals will populate the known bound singleparticle states in ${ }^{133} \mathrm{Sn}$, which decay via $\gamma$-ray emission to the ground state. Consequently, the 513-, 854-, 1561-, and $2002-\mathrm{keV}$ transitions are all clearly visible in the spectrum shown in Fig. 1. With respect to the yet unknown position of the $0 i_{13 / 2}$ orbital we note that in the present experiment no $\gamma$ ray is observed in the expected energy range $\left(E_{x}=2360-2600 \mathrm{keV}[12]\right)$.

Besides the removal of one of the two valence neutrons also the knockout of a neutron from the closed $N=82$ core can occur. In this case neutron-hole states in ${ }^{133} \mathrm{Sn}$ are populated as illustrated in Fig. 3(a). Although knockout can proceed from all five orbitals of the $N=50-82$ shell, the largest cross section is expected for the $0 h_{11 / 2}$ orbital since it is close to the Fermi level and occupied by as many as twelve neutrons. We therefore suggest that the 3570(50)$\mathrm{keV}$ transition corresponds to the decay of a $11 / 2^{-}$state to

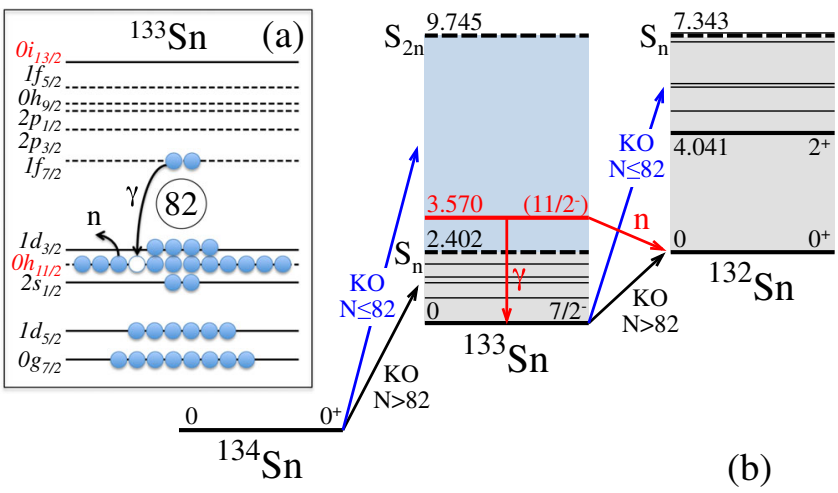

FIG. 3. (a) Schematic of the neutron orbital occupancies after the knockout of a $0 h_{11 / 2}$ neutron from the ${ }^{134} \mathrm{Sn}$ projectile, followed by $\gamma$ decay or neutron emission; (b) Illustration of the population of bound (gray regions) and unbound (blue region) excited states in ${ }^{132,133} \mathrm{Sn}$ following one-neutron knockout (KO) from either the valence space $(N>82)$ or the $N=50-82$ core $(N \leq 82)$. Energies are quoted in $\mathrm{MeV}$. 
the ground state. Note that this energy is close to the 3.66 MeV proposed in Ref. [8]. Since this excited state lies above the neutron-separation energy, it can decay either via neutron emission or electromagnetic decay; see Fig. 3(b). Because of the high excitation energy of the first excited state in ${ }^{132} \mathrm{Sn}, E_{x}\left(2^{+}\right)=4.041 \mathrm{MeV}$, the presumed $11 / 2^{-}$ state at $3570(50) \mathrm{keV}$ can only neutron decay to the ground state of ${ }^{132} \mathrm{Sn}$, i.e., via the emission of an $\ell=5$ neutron with a kinetic energy of about $1.2 \mathrm{MeV}\left(E_{n}=E_{x}-S_{n}\right)$. The expected lifetime for this decay amounts to less than $10^{-17} \mathrm{~s}$. To estimate the lifetime for an $E 2 \gamma$ decay to the $7 / 2^{-}$ground state, we assume a transition strength of 2 W.u. in line with the experimental strength of several single-particle E2 transitions in ${ }^{133} \mathrm{Sn}$ and ${ }^{209} \mathrm{~Pb}$ as discussed above. This estimate yields a lifetime of about $10^{-14} \mathrm{~s}$. Therefore, at the level of pure single-particle transitions, the neutron decay is expected to be roughly 3 orders of magnitude faster as compared to $\gamma$-ray emission.

As mentioned above, an additional factor, which can affect the competition between neutron and $\gamma$-ray emission, is the overlap of the wave functions of the initial and final states. After the knockout of a core neutron, e.g., a $0 h_{11 / 2}$ neutron, from the projectile ${ }^{134} \mathrm{Sn},{ }^{133} \mathrm{Sn}$ is populated with a neutron configuration as shown in Fig. 3(a). In the case of $\gamma$ decay, the hole in the $N=50-82$ core is filled by one of the two neutrons occupying primarily the $1 f_{7 / 2}$ orbital in the ground state of ${ }^{134} \mathrm{Sn}$ and a $3570-\mathrm{keV} \gamma$ ray is emitted. The final state corresponds to the ground state of ${ }^{133} \mathrm{Sn}$. Neutron emission, on the other hand, yields a ${ }^{132} \mathrm{Sn}$ nucleus with two holes in the $0 h_{11 / 2}$ orbital and two neutrons above the $N=82$ gap, i.e., in a two-particle-two-hole $(2 p-2 h)$ state. The ground state of ${ }^{132} \mathrm{Sn}$, however, is not expected to contain large contributions of $2 p-2 h$ configurations and, consequently, the overlap of the wave function of the parent state with that of the daughter state plus a neutron is small. It is this wave function mismatch which hinders neutron emission and allows electromagnetic decay to compete in the decay of highly excited neutron-hole states in ${ }^{133} \mathrm{Sn}$. Note that the above reasoning is valid for all hole states in the $N=50-82$ shell, not only the $0 h_{11 / 2}$ hole.

In order to quantify the average $\gamma$-ray branching for states above the neutron-separation energy in ${ }^{133} \mathrm{Sn}$, we will discuss in the following the total cross sections for oneneutron knockout from ${ }^{133} \mathrm{Sn}$ and ${ }^{134} \mathrm{Sn}$ projectiles, $\sigma_{1 n}$, which are shown together with the cross sections for multineutron removal $\sigma_{x n}$ in Fig. 4. The measured cross sections, $\sigma_{1 n}=183(21) \mathrm{mb}$ for ${ }^{133} \mathrm{Sn}$ and $\sigma_{1 n}=$ $69(10) \mathrm{mb}$ for ${ }^{134} \mathrm{Sn}$, comprise contributions from both the removal of a neutron from the valence space $(N>82)$ $\sigma_{1 n}^{\mathrm{val}}$, as well as knockout from the $N=50-82$ core, $\sigma_{1 n}^{\text {core }}$, as discussed above. These contributions were calculated using eikonal reaction theory $[20,21]$ and employing the ground state wave functions from the shell model, the known SPE in ${ }^{133} \mathrm{Sn}$ and excitation energies for the neutron-hole states from a spherical Hartree-Fock (HF) calculation. These calculations yield values of $\sigma_{1 n}^{\mathrm{val}} \sim 14 / 20$ and $\sigma_{1 n}^{\text {core }} \sim$ $186 / 152 \mathrm{mb}$ for knockout from ${ }^{133} \mathrm{Sn} /{ }^{134} \mathrm{Sn}$. For removal from ${ }^{133} \mathrm{Sn}$ it is expected that due to the high neutronseparation energy in the daughter nucleus ${ }^{132} \mathrm{Sn}, S_{n}=$ 7.343(7) MeV [13], most of the highly excited states populated following the removal of a neutron from the $N=$ 50-82 core are bound and decay via $\gamma$-ray emission as illustrated in Fig. 3(b). Indeed, the calculated value, $\sigma_{1 n}=\sigma_{1 n}^{\text {val }}+\sigma_{1 n}^{\text {core }} \sim 200 \mathrm{mb}$, is in good agreement with the measured cross section. In the case of knockout from ${ }^{134} \mathrm{Sn}$, in contrast, the residual nucleus ${ }^{133} \mathrm{Sn}$ has a low neutron-separation energy of only $S_{n}=2.402(4) \mathrm{MeV}$ [13] and, as a consequence, neutron removal from the core populates unbound states which can decay either via neutron or $\gamma$-ray emission. In the case of neutron emission the final nucleus is identified as ${ }^{132} \mathrm{Sn}$ in the $\mathrm{ZD}$ spectrometer and the corresponding cross section is, therefore, assigned to the two-neutron removal reaction. As a consequence the measured value of $\sigma_{2 n}$ is larger as compared to $\sigma_{1 n}$ (see Fig. 4). The cross section corresponding to $\gamma$-decaying unbound states in ${ }^{133} \mathrm{Sn}$ can be estimated by subtracting the calculated cross section for neutron removal from the valence space to bound final states in ${ }^{133} \mathrm{Sn}$, amounting to $\sigma_{1 n}^{\mathrm{val}} \sim 20 \mathrm{mb}$, from the measured $\sigma_{1 n}=69(10) \mathrm{mb}$. The resulting value of $\sim 49 \mathrm{mb}$, compared to the calculated value of $\sigma_{1 n}^{\text {core }} \sim 152 \mathrm{mb}$ for knockout from the core, suggests that $25 \%-35 \%$ of the decay of unbound states in ${ }^{133} \mathrm{Sn}$, populated in the one-neutron knockout reaction, proceeds via $\gamma$-ray emission. Although this estimate is subject to several uncertainties it clearly shows that the contribution of $\gamma$ decay is significant.

The effect of nuclear structure on the competition between neutron and $\gamma$ decay of unbound states may have

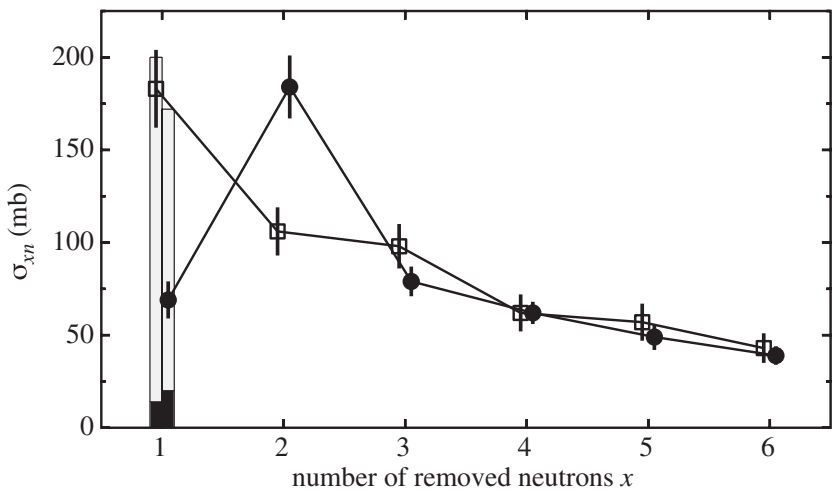

FIG. 4. Total experimental cross sections for the removal of $x$ neutrons, $\sigma_{x n}$, from ${ }^{133} \mathrm{Sn}$ (open squares) and ${ }^{134} \mathrm{Sn}$ (filled circles) projectiles. Lines are drawn to guide the eye. The vertical bars for $x=1$ indicate the cross sections for knockout from the $N>82$ valence space (black) and from the $N=50-82$ core (gray) as calculated using eikonal reaction theory. The contribution from the $0 h_{11 / 2}$ orbital to the latter amounts to 67 and $56 \mathrm{mb}$, respectively. 
more general and further-reaching consequences. The $\beta$ decay properties of nuclei in the region southeast of ${ }^{132} \mathrm{Sn}$ are of great importance for the description of the $r$ process of nuclear synthesis $[22,23]$. In this region, the $\beta$-decay energies $Q_{\beta}$ are large and the neutron-separation energies $S_{n}$ low, so that unbound excited states in a wide energy window of more than $10 \mathrm{MeV}$ can be populated in the $\beta$ decays. Experimental information is scarce so that in many cases theoretical calculations of global $\beta$-decay properties have to be relied upon. In the latter $\beta$-delayed neutron emission probabilities are deduced from calculated strength functions under the assumption that neutron emission occurs whenever it is energetically possible [see, for example, Eq. (21) of Ref. [24]]. It is well known that for nuclei with $Z<50$ and $N \approx 82$, the $\beta$ decay is dominated by the $\nu 0 g_{7 / 2} \rightarrow \pi 0 g_{9 / 2}$ Gamow-Teller transition [24,25]. Also the first-forbidden $\nu 0 h_{11 / 2} \rightarrow \pi 0 g_{9 / 2}$ decay is known to play a significant role. Beyond $N=82$, once the $1 f_{7 / 2}$ neutron orbital starts being occupied, both these transitions populate core-excited states at high excitation energy in the daughter nuclei. In ${ }^{132} \mathrm{Sn}$ and ${ }^{131}$ In, populated in the $\beta$ decays of the $N=83$ isotones ${ }^{132} \mathrm{In}$ [26] and ${ }^{131} \mathrm{Cd}$ [27], excited states comprising a neutron hole in the $0 h_{11 / 2}\left(0 g_{7 / 2}\right)$ orbital have been identified in the energy range of 4-5 MeV (6-7 MeV). For the decay of these states, the same structure arguments apply, which have been put forward above in the discussion of the decay of highly excited neutron-hole states in ${ }^{133} \mathrm{Sn}$. It therefore seems advisable to fully elucidate the neutron versus $\gamma$-ray competition in the decay of unbound excited states in exotic nuclei southeast of ${ }^{132} \mathrm{Sn}$ in future experiments.

To conclude, we presented clear evidence for the electromagnetic decay of states in ${ }^{133} \mathrm{Sn}$ at excitation energies up to more than $3 \mathrm{MeV}$ above the neutronseparation energy. These excited states are interpreted as neutron-hole states that are populated following the knockout of a neutron from the closed $N=50-82$ shell of the ${ }^{134} \mathrm{Sn}$ projectile ion at relativistic energies. The ability of $\gamma$ ray emission to compete with neutron decay, despite a hindrance of 3 orders of magnitude at the single-particle level, is explained taking into account the structure of the initial and final states and the resultant wave-function overlap. Our study raises the question whether, due to nuclear structure effects, the $\gamma$-ray emission may play a much more significant role than generally assumed in the decay of highly excited states populated following $\beta$ decay in the region southeast of ${ }^{132} \mathrm{Sn}$.

A. J. acknowledges fruitful discussions with $\mathrm{H}$. Grawe, S. L. Tabor, and J.L. Tain. We thank the staff of the RIKEN Nishina Center accelerator complex for providing stable beams with high intensities to the experiment. This work was supported by the Spanish Ministerio de
Ciencia e Innovación under Contract No. FPA2011-29854C04 and the Spanish Ministerio de Economía y Competitividad under Contract No. FPA2014-57196-C54-P. J. A. T. acknowledges the support of the Science and Technology Facilities Council (UK) Grant No. ST/ L005743 and R. O. that of JSPS KAKENHI Grant No. 26887048. G. M. T. gratefully acknowledges funding of this research from the Research Council of Norway, Project Grant No. 222287.

*andrea.jungclaus@csic.es

[1] E. Fermi, Nuclear Physics (University of Chicago Press, Chicago, 1950).

[2] S. R. Stroberg et al., Phys. Rev. C 90, 034301 (2014).

[3] J. L. Tain et al., Phys. Rev. Lett. 115, 062502 (2015).

[4] R. Dungan et al., Phys. Rev. C 93, 021302(R) (2016).

[5] A. Spyrou et al., Phys. Rev. Lett. 117, 142701 (2016).

[6] X. Xu et al., Phys. Rev. Lett. 117, 182503 (2016).

[7] S. E. A. Orrigo et al., Phys. Rev. C 93, 044336 (2016).

[8] P. Hoff et al., Phys. Rev. Lett. 77, 1020 (1996).

[9] K. L. Jones et al., Nature (London) 465, 454 (2010).

[10] K. L. Jones et al., Phys. Rev. C 84, 034601 (2011).

[11] J. M. Allmond et al., Phys. Rev. Lett. 112, 172701 (2014).

[12] W. Reviol et al., Phys. Rev. C 94, 034309 (2016).

[13] M. Wang, G. Audi, A. H. Wapstra, F. G. Kondev, M. MacCormick, X. Xu, and B. Pfeiffer, Chin. Phys. C 36, 1603 (2012).

[14] T. Kubo et al., Prog. Theor. Exp. Phys. 2012, 3C003 (2012).

[15] N. Fukuda, T. Kubo, T. Ohnishi, N. Inabe, H. Takeda, D. Kameda, and H. Suzuki, Nucl. Instrum. Methods Phys. Res., Sect. B 317, 323 (2013).

[16] S. Takeuchi, T. Motobayashi, Y. Togano, M. Matsushita, N. Aoi, K. Demichi, H. Hasegawa, and H. Murakami, Nucl. Instrum. Methods Phys. Res., Sect. A 763, 596 (2014).

[17] S. Agostinelli et al., Nucl. Instrum. Methods Phys. Res., Sect. A 506, 250 (2003).

[18] http://www.nndc.bnl.gov/ensdf/, updated evaluation of data for ${ }^{209} \mathrm{~Pb}$ by F.G. Kondev ENSDF 14 June 2015.

[19] L. Coraggio, A. Covello, A. Gargano, and N. Itaco, Phys. Rev. C 88, 041304(R) (2013), and references therein.

[20] J. A. Tostevin, Nucl. Phys. A682, 320 (2001).

[21] P. G. Hansen and J. A. Tostevin, Annu. Rev. Nucl. Part. Sci. 53, 219 (2003).

[22] G. Lorusso et al., Phys. Rev. Lett. 114, 192501 (2015).

[23] M. R. Mumpower, R. Surman, G. C. McLaughlin, and A. Aprahamian, Prog. Part. Nucl. Phys. 86, 86 (2016).

[24] T. Marketin, L. Huther, and G. Martínez-Pinedo, Phys. Rev. C 93, 025805 (2016).

[25] I. N. Borzov, J. J. Cuenca-García, K. Langanke, G. Martínez-Pinedo, and F. Montes, Nucl. Phys. A814, 159 (2008).

[26] B. Fogelberg, M. Hellstrom, D. Jerrestam, H. Mach, J. Blomqvist, A. Kerek, L. O. Norlin, and J. P. Omtvedt, Phys. Rev. Lett. 73, 2413 (1994).

[27] J. Taprogge et al., Eur. Phys. J. A 52, 347 (2016). 